\title{
Pressure and frequency dependent linkage between motility and epithelial secretion in human proximal small intestine
}

\author{
A Mellander, K Järbur, H Sjövall
}

\begin{abstract}
Background-Motor disturbances are sometimes associated with diarrhoea by unknown mechanisms.

Aim-To determine if there is a quantitative link between intestinal motility and epithelial secretion.

Subjects-Experiments were performed in 21 healthy volunteers and three patients with villus atrophy.

Methods-Duodenal and jejunal motor activities were registered in the fasted state by open tip manometry. Secretion was measured directly by marker perfusion and indirectly by recording transmural potential difference (PD).

Results-A significant correlation was found between "low pass filtered" pressure and PD, but no correlation was found between amplitudes of isolated contractions and PD changes. During repeated phasic contractions (phase III of migrating motor complex), PD increased at a rate that was higher in the duodenum than in the jejunum, and higher in patients with villus atrophy than in healthy controls. After reaching a peak, PD decreased despite continuing phasic motor activity, provided that there was no concomitant increase in mean pressure. Fluid secretion increased roughly in parallel with $P D$, except at the very end of the cycle.

Conclusions-To explain these findings, one has to postulate participation of at least two types of receptor: a slowly adapting pressure sensitive receptor and another mechanoreceptor, possibly a mucosal touch receptor, to account for the run down phenomenon. This model predicts that short lasting trains of contractions, so called discrete clusters, will be a particularly potent stimulus for activation of mucosal secretion.

(Gut 2000;46:376-384)
\end{abstract}

Internal Medicine,

University of

Göteborg, Sweden

A Mellander

K Järbur

H Sjövall

Correspondence to: Dr H Sjövall, Department of Internal Medicine, Sahlgrenska University

Hospital, S-413 45

Göteborg, Sweden

Accepted for publication 14 October 1999

Keywords: enteric nervous system; intestinal mucosa; migrating motor complex; receptor; chemoreceptor; bile

There is strong evidence for an interaction between intestinal motility and epithelial secretion, ${ }^{1-3}$ but the mechanism is not altogether understood. The linkage is probably mediated by the enteric nervous system, ${ }^{4-5}$ and the effector mechanism seems to be opening of the cystic fibrosis transmembrane conductance regulator. ${ }^{6}$ The association phenomenon is resistant to atropine, ${ }^{7}$ extrinsic denervation of the segment, ${ }^{48}$ selective damage to the myenteric plexus, ${ }^{8}$ and villus atrophy caused by coeliac disease. ${ }^{39}$ To account for all these findings, one has to postulate that the entire circuitry, including the receptor, is confined to the mucosa and the submucous ganglia. A similar reflex seems to exist also in the proximal duodenum ${ }^{10}$ and the distal colon. ${ }^{11-14}$

The physiological and pathophysiological role of this linkage mechanism is not known. Various "pathological" motor patterns have been described in severe dyspepsia, ${ }^{15}$ irritable bowel syndrome, ${ }^{16}$ and intestinal pseudoobstruction, ${ }^{17}$ but it is not known how these patterns affect intestinal epithelial function. The linkage mechanism may be mediated by mucosal touch receptors ${ }^{18}$ or myenteric stretch/ tension receptors, ${ }^{19}$ and may at least be modulated also by luminal chemoreceptors. ${ }^{20}$ Previous studies of pressure-transmural potential difference (PD) linkage have mainly been descriptive, and no attempt has been made to quantify the linkage function in humans. It has also been assumed that the PD increase that occurs during periods of intense motility is accompanied by net fluid secretion, but this has not yet been confirmed. In fact, it can by no means be excluded that intense motor activity may also affect electrically silent sodium absorption, either through neural mechanisms ${ }^{21}$ or by increasing the functional surface area, for example. ${ }^{22}$

In the experiments described below, we tried to quantify the relation between interdigestive motility and epithelial function. By analysing in detail how the linkage mechanism responds to changes in pressure and frequency, we hoped to draw conclusions about the type of receptor involved. This type of information should make it possible to predict and understand the secretory consequences of normal and pathological intestinal motor patterns.

Materials and methods
Abbreviations used in this paper: $M M C$, migrating motor complex; NFT, net fluid transport; PD, transmural potential difference; PEG, poly(ethylene glycol). 
obtained during gastroscopy, as part of a diagnostic work up for suspected coeliac disease. ${ }^{9}$ The experimental protocol was approved by the ethical committee at Sahlgrenska University Hospital, Göteborg, and all participants had given informed consent.

GENERAL DESIGN OF THE STUDY

Two series of experiments were performed. In the first series $(n=17)$, we studied the quantitative linkage between pressure waves and corresponding changes in PD. In most of these experiments, recordings were made from both distal duodenum and proximal jejunum. In the second series ( $n=21)$, we used a perfusionaspiration technique to measure simultaneously motor activity, PD, bile release, and net fluid transport (NFT) in the distal duodenum.

After fasting overnight, the subjects came to the laboratory at $7.30 \mathrm{am}$, and were then transnasally intubated with a multilumen catheter. The correct position of the tube was checked by intermittent fluoroscopy. The total duration of the experiment was about four hours.

DIGITAL SYSTEM FOR RECORDING OF

INTRALUMINAL PRESSURE AND TRANSMURAL PD In the first series, we used a commercially available computerised system (Synectics Medical, Stockholm, Sweden) for simultaneous recordings of interdigestive motility and PD. The principle behind the set up is that a saline infusion is used as a flowing electrode, and the potential difference between the infused solution and the subcutaneous compartment is measured with calomel half cells. ${ }^{23}$ The intestinal multilumen catheter (Arndorfer Inc, Greendale, Wisconsin, USA) had an outer diameter of $4.6 \mathrm{~mm}$, a central lumen of $1.8 \mathrm{~mm}$ for a guide wire, and three channels with a diameter of $0.8 \mathrm{~mm}$ ending 2,17 , and $32 \mathrm{~cm}$ from the tip of the tube. These three recording side ports were placed in the proximal jejunum, at the duodenojejunal flexure, and in the midduodenum respectively. Each channel was perfused separately with isotonic saline at a rate of $0.3 \mathrm{ml} / \mathrm{min}$ through narrow capillaries under low compliance conditions. Inflow pressure was recorded by pressure transducers connected to a polygraph (PC Polygraph; Synectics), which converted the voltage signal into digital information at $4 \mathrm{~Hz}$. PD was recorded in the mid-duodenum and the proximal jejunum by calomel half cells (K 401; Radiometer, Copenhagen, Denmark) connected to the infusion lines by salt bridges. The reference electrode consisted of a saline filled plastic cannula (Venflon; outer diameter $1.0 \mathrm{~mm}$; Viggo-Spectramed, Helsingborg, Sweden) inserted subcutaneously into the left forearm. The voltage signal was filtered and processed by a specially constructed high impedance amplifier, and the data were transferred from the polygraph to an IBM compatible computer through a fibreoptic interface. The individual recording was displayed on the computer screen during the experiment and was stored on the hard disk for later analysis. Mean values for intraluminal pressure and transmural PD were calculated by the software of the supplier (Polygram 5; Synectics).

DATA ANALYSIS AND EXPRESSION OF RESULTS Three specific aspects of the motility-PD linkage were analysed: (a) the relation between the amplitude of isolated contractions and concomitant PD changes; $(b)$ the relation between "low pass filtered" slow changes in pressure and PD; (c) PD changes occurring during sustained phasic motor activity at slow wave frequency (migrating motor complex (MMC) phase III).

(a) The analysis of the relation between the amplitude of isolated contractions and concomitant PD changes was performed in a randomly chosen subgroup $(\mathrm{n}=5)$ in which all isolated contractions were studied $(\mathrm{n}=47)$. Isolated contractions were defined in this context as those with an amplitude of $15 \mathrm{~mm} \mathrm{Hg}$ or more, preceded and followed by motor quiescence for at least 60 seconds. The onset PD was defined as the PD value before the start of the contraction. A contraction related PD increase was defined as an increase in PD that started before the end of the individual contraction. The amplitude of this PD wave was defined as the maximal increase in PD attained in association with the wave.

(b) The pressure response to a contraction wave is virtually instantaneous, but the PD response is slower, being limited by the reactivity of the epithelium. To compare quantitatively pressure and PD changes under similar time resolution conditions, the two signals were low pass filtered in the following way: mean pressure and PD values were first read at 30 second intervals, from the original curve. Each consecutive pair of data was then replaced with their mean, and this procedure was repeated twice, on the calculated mean values - that is, each final value was to some degree influenced by the original 30 second data from a four minute period. This procedure thus "blunts" short lasting phasic events, but has only a minor influence on slow steady changes in the signal, an effect similar to low pass filtration of the data.

(c) PD changes before, during, and after phase III were studied in control subjects (duodenum and jejunum) and also in the three patients with villus atrophy (duodenum only). Pressure and PD during phase III were analysed at 10 second intervals. The onset of phase III activity was defined as the first 10 second interval of the period fulfilling phase III criteria. The onset of the phase III associated PD rise was defined as the first time point in the first series of five sequential PD increases. The peak values for pressure and PD were defined as the maximal value recorded during phase III activity. The initial slope of the rising phase of the PD curve was calculated by linear regression from the first five 10 second points, starting from the onset of the PD curve.

When comparing the pressure and PD pattern in the duodenum and jejunum, we studied three defined periods: phase III, phase I-II (first 30 minutes of cycle), and late phase II (last 30 minutes of cycle). During phase III, we 
also measured peak values, the time required to reach peak values, and the mean upward slope of the PD curve. In contrast with the initial slope, which was based on the first five $10 \mathrm{sec}-$ ond values, the mean slope was calculated by linear regression from all data points between onset and peak value. The reason for the different mode of calculation was that the start of the jejunal PD increase was sometimes less distinct than in the duodenum, resulting in the risk of a large error if the slope was extrapolated from only a few initial values. To evaluate if the slope and maximal value of the phase III associated PD curve was related to mucosal secretory function, we also measured these variables in three patients with hypersecretion resulting from villus atrophy. Altogether eight phase III periods were obtained in these experiments.

TECHNIQUE FOR MEASUREMENT OF BILE RELEASE AND NET FLUID TRANSPORT (SECOND SERIES)

To quantify these parameters, we used a modified triple lumen perfusion technique that has been described in detail elsewhere. ${ }^{79}$ Briefly, a specially designed tube containing one infusion channel, two aspiration channels, and two pressure/PD channels was placed with its tip at the duodenojejunal flexure. An isotonic electrolyte solution (composition in $\mathrm{mmol} / \mathrm{l}: 122$ $\mathrm{NaCl}, 4.7 \mathrm{KCl}, 1.2 \mathrm{KH}_{2} \mathrm{PO}_{4}, 25 \mathrm{NaHCO}_{3}, 1.2$ $\mathrm{MgCl}_{2}, 30$ mannitol) was preheated to $37^{\circ} \mathrm{C}$ and infused through the most proximal channel ending just distal to the papilla Vateri. The solution also contained poly(ethylene glycol) (molecular mass $4000 \mathrm{Da}$ ) at a concentration of $2 \mathrm{~g} / 1$ as a non-absorbable volume marker. The reason for using the Krebs/mannitol solution is that mannitol can be conveniently replaced with glucose without changing the osmolarity or electrolyte contents. The infusion rate was about $6 \mathrm{ml} / \mathrm{min}$, the exact rate being determined in each experiment. After a 40 minute equilibration period, fluid was aspirated manually in 15 minute aliquots through the two aspiration channels ending $5 \mathrm{~cm}$ ("proximal aspiration") and $15 \mathrm{~cm}$ ("distal aspiration") distal to the infusion site. The distal aspirate was collected five minutes after the proximal aspirate to compensate for the mean transit time in the test segment. The concentration of PEG in the proximal and distal aspirate was determined turbidimetrically. ${ }^{23}$ The concentration of bilirubin in the proximal aspirate was measured by a technique based on the accelerated diazo reaction. ${ }^{7}$

Bilirubin release $\left(\mathrm{Q}_{\mathrm{bil}}\right)$ was calculated from the total volume flow and the bilirubin concentration ([bilirubin]) at the proximal aspiration site: $\mathrm{Q}_{\text {bil }}=[$ bilirubin $] \times$ pump rate $\times\left[\mathrm{PEG}_{\text {inf }}\right] /$ $\left[\mathrm{PEG}_{\mathrm{pr}}\right]$

NFT in the $10 \mathrm{~cm}$ test segment was calculated from the flow volume entering the test segment $\left(\mathrm{Q}_{\mathrm{pr}}\right)$ minus the proximal aspiration rate $(0.7 \mathrm{ml} / \mathrm{min})$, and the dilution of PEG between the proximal and distal aspiration sites according to the following equation: NFT = $\left(\mathrm{Q}_{\mathrm{pr}}-0.7\right) \times\left(1-\left[\mathrm{PEG}_{\mathrm{pr}}\right] /\left[\mathrm{PEG}_{\mathrm{di}}\right]\right)$

where $\mathrm{Q}_{\mathrm{pr}}=$ pump rate $\times\left[\mathrm{PEG}_{\mathrm{inf}}\right] /\left[\mathrm{PEG}_{\mathrm{pr}}\right]$ and $\left[\mathrm{PEG}_{\mathrm{inf}}\right]$ is the PEG concentration in infused solution $(=2 \mathrm{~g} / \mathrm{l}),\left[\mathrm{PEG}_{\mathrm{pr}}\right]$ is the PEG concen- tration in proximal aspirate, and $\left[\mathrm{PEG}_{\mathrm{di}}\right]$ is the PEG concentration in distal aspirate.

Intestinal motor activity was measured by a pneumohydraulic system similar to that described above (modified from that of Arndorfer and colleagues $\left.{ }^{24}\right)$. Sterile water or isotonic saline (for PD recording) was infused at a rate of $0.3 \mathrm{ml} / \mathrm{min}$ through two separate polyethyl-

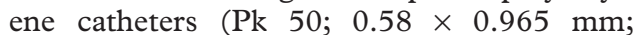
Meteko Instrument AB, Hägersten, Sweden) ending $2 \mathrm{~cm}$ distal to the infusion site and at the tip of the tube. Infusion pressure was continuously recorded by connecting the infusion lines to Statham pressure transducers $(23 \mathrm{DC}$; Statham Instruments, Oxnard, California, USA), and the signal was displayed on a Grass polygraph (model 7D; Grass Instruments, Quincy, Massachusetts, USA). A contraction was defined as a phasic pressure wave with an amplitude of at least $8 \mathrm{~mm} \mathrm{Hg}$, and the contraction frequency-that is, numbers of contractions per minute-was calculated manually from the paper recordings.

DATA ANALYSIS AND EXPRESSION OF RESULTS The duration of a full MMC cycle (defined as the time between the end of two consecutive phase III periods, see below) normally varies considerably both between and within subjects. ${ }^{25}{ }^{26}$ To study transport as a function of time in groups of subjects, one has to generate artificially a common time scale. This was achieved by dividing each MMC cycle into 20 equal-that is, 5\% - time intervals, each interval being represented by the corresponding values for NFT, bile release, and the mean contraction frequency recorded during the same (15 minute) time period. NFT, bile release, and contraction frequency were then plotted against a common time scale expressed as percentage of the full cycle length. ${ }^{27}$ The frequency recording (which was read at one minute intervals) was thus deliberately "blunted" to generate the same time resolution as in the NFT measurements.

ANALYSIS OF COVARIATION BETWEEN MOTILITY, BILE RELEASE, AND NFT

The bilirubin concentrations in particular varied considerably both within and between subjects, and we therefore considered parametric tests based on confidence intervals to be inappropriate. The variability was, however, much smaller at the start of the MMC cycle. We therefore first measured mean transport rate at its highest value (maximal absorption), which occurred in the $20-25 \%$ interval of the cycle. We then tested the probability that a 2 SD change in transport rate in the secretory direction was predicted by either a $2 \mathrm{SD}$ increase in motor activity or a 2 SD increase in bile release, or a combination of the two. The material for this calculation consisted of 13 full MMC cycles.

COMMENTS ON POLARITY OF PD SIGNAL

The polarity and magnitude of the PD signal depend on the perfusate. With our Krebs/ mannitol perfusate, the "basal" PD is usually 
slightly lumen negative $(0-2 \mathrm{mV})$ and it becomes more lumen negative during increased motor activity. To avoid confusion, the sign of the PD signal consistently refers to the luminal polarity - that is, PD is generally given a negative sign.

DEFINITIONS OF MMC PERIODS

These were as follows:

- phase I: motor quiescence (two contractions/10 min, or less) after a phase III period;

- phase II: periods not fulfilling phase I or phase III criteria;

- phase III: propagating maximal motor activity at slow wave frequency $(10-12 / \mathrm{min}$ in the human proximal small intestine) with a duration of at least two minutes;

- phase I-II: first 30 minutes after, but not including, phase III;

- late phase II: last 30 minutes preceding, but not including, phase III;

- full MMC cycle: time between two consecutive phase III periods.

STATISTICAL ANALYSIS

All results are expressed as mean (SE). In the statistical analysis of the relation between contraction frequency, NFT, and transmural PD, we used a non-parametric analysis of variance for repeated measurements (Friedmans $F$ test). In the analysis of the relation between mean pressure and PD during the whole MMC cycle, we first low pass filtered the dynamic pressure and PD changes as described above. The resulting values were then plotted in an $x-y$ diagram, and the significance of the slope of the resulting regression line was tested by linear regression analysis and analysis of variance. The significance of differences between groups was tested with the non-parametric Wilcoxon test and the Mann-Whitney U test. $\mathrm{p}<0.05$ was regarded as statistically significant.

\section{Results}

QUANTITATIVE ANALYSIS OF PRESSURE-PD RELATION

Analysis of individual contractions

The relation between the contraction amplitudes and the resulting PD changes was studied in a randomly chosen subgroup of subjects $(n=5)$, in which all isolated contractions (for definition, see methods) exceeding $15 \mathrm{~mm}$ $\mathrm{Hg}$ were measured and analysed. Table 1 summarises the data. Isolated phasic contractions always resulted in a more lumen negative PD, but in the whole material $(n=47)$, there was no significant correlation between the amplitude of individual contractions and the associated

Table 1 Amplitudes and corresponding transmural potential difference (PD) deflections of 47 isolated contractions with an amplitude exceeding $15 \mathrm{~mm} \mathrm{Hg}$ in five subjects

\begin{tabular}{lllll}
\hline MMC period & $n$ & $\begin{array}{l}\text { Contraction } \\
\text { amplitude }\end{array}$ & PD deflection & Ratio \\
\hline Phase I-II & 17 & $26(3)$ & $-0.22(0.04)$ & $-8.5(1.7)$ \\
Late phase II & 30 & $37(3)^{\star}$ & $-0.43(0.06)^{\star}$ & $-13.9(2.0)$ \\
All & 47 & $33(2)$ & $-0.36(0.04)$ & $-11.9(1.5)$ \\
\hline
\end{tabular}

See Materials and Methods for definitions. Contraction amplitudes are given in $\mathrm{mm} \mathrm{Hg}, \mathrm{PD}$ deflections in $\mathrm{mV}$ (lumen negative polarity), and the ratios as $\mu \mathrm{V} / \mathrm{mm} \mathrm{Hg}$. Values are mean (SEM). ${ }^{\star} \mathrm{p}<0.05$, significant difference between migrating motor complex (MMC) periods.

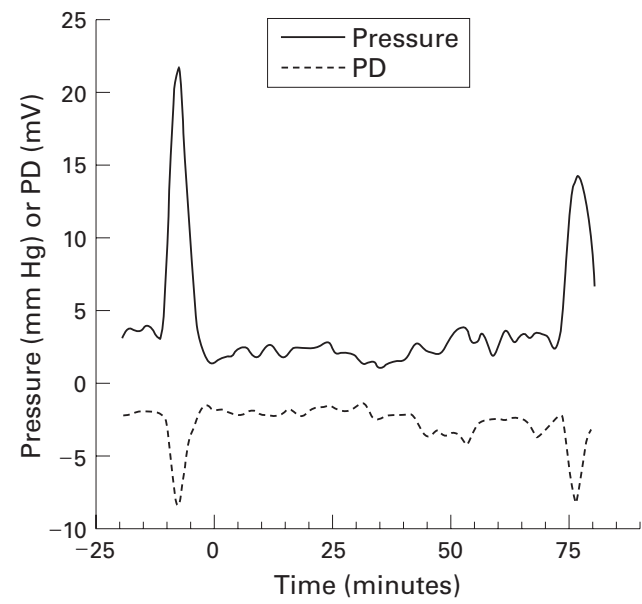

Figure 1 An example of low pass filtered pressure and transmural potential difference (PD) data from a full migrating motor complex cycle, in a healthy volunteer. Pressure is given in $\mathrm{mm} \mathrm{Hg}$ and PD in $\mathrm{mV}$ (luminal polarity). The time scale is in minutes, and time zero is the end of the first duodenal phase III period.

changes in PD $\left(r^{2}=0.058\right)$. The mean amplitude of the PD deflection induced by a single contraction in the whole material was $0.36(0.04) \mathrm{mV}$. Both contraction amplitudes and the amplitude of the PD deflections were significantly lower during the first part of the cycle. The "gain" of the response-that is, the increase in PD per unit pressure increasetended to be higher at the end of the cycle (phase I-II: - 8.5 (1.7) $\mu \mathrm{V} / \mathrm{mm} \mathrm{Hg}$; late phase II: - $13.9(2.0) \mu \mathrm{V} / \mathrm{mm} \mathrm{Hg})$, but this difference did not reach significance (Mann-Whitney $U$ test, $p=0.08$ ).

Relation between slow changes in pressure and PD during a full MMC cycle

During the entire MMC cycle except in late phase III, pressure and PD changed approximately in parallel. The maximal rate of increase in PD is obviously limited by the responsiveness of the epithelium - that is, a relatively slow process. To eliminate the impact of this phenomenon, we low pass filtered both the pressure and PD curves, using the mode of recalculation described in the methodological section. This was performed in the seven experiments in which we registered a full MMC cycle with the digital recording system. Figure 1 gives an example of low pass filtered pressure and PD signals from one subject. It can be seen that the pressure and PD curves are essentially mirror images of each other. This phenomenon was quantified by plotting the low pass filtered pressure and PD data against each other. Figure 2 shows the resulting plot from the same subject. Similar results were obtained in the other subjects: the correlation between pressure and PD was in all cases highly significant $(r=0.84(0.03)$; range $0.70-$ $0.94)$. The mean slope of the regression lines was $-0.33(0.07) \mathrm{mV} / \mathrm{mm} \mathrm{Hg}$ (range -0.15 to $-0.71 \mathrm{mV} / \mathrm{mm} \mathrm{Hg}$ ) - that is, when low pass filtered pressure increased by $1 \mathrm{~mm} \mathrm{Hg}$, the mean PD became 0.33 (0.07) $\mathrm{mV}$ more lumen negative. 


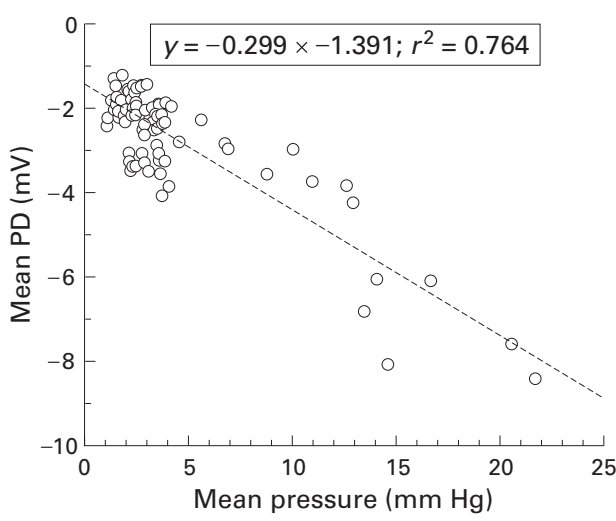

Figure 2 Correlation between pressure and transmural potential difference (PD) data from the same subject as in fig 1. The slope of the regression line was highly significant $(p<0.001)$.

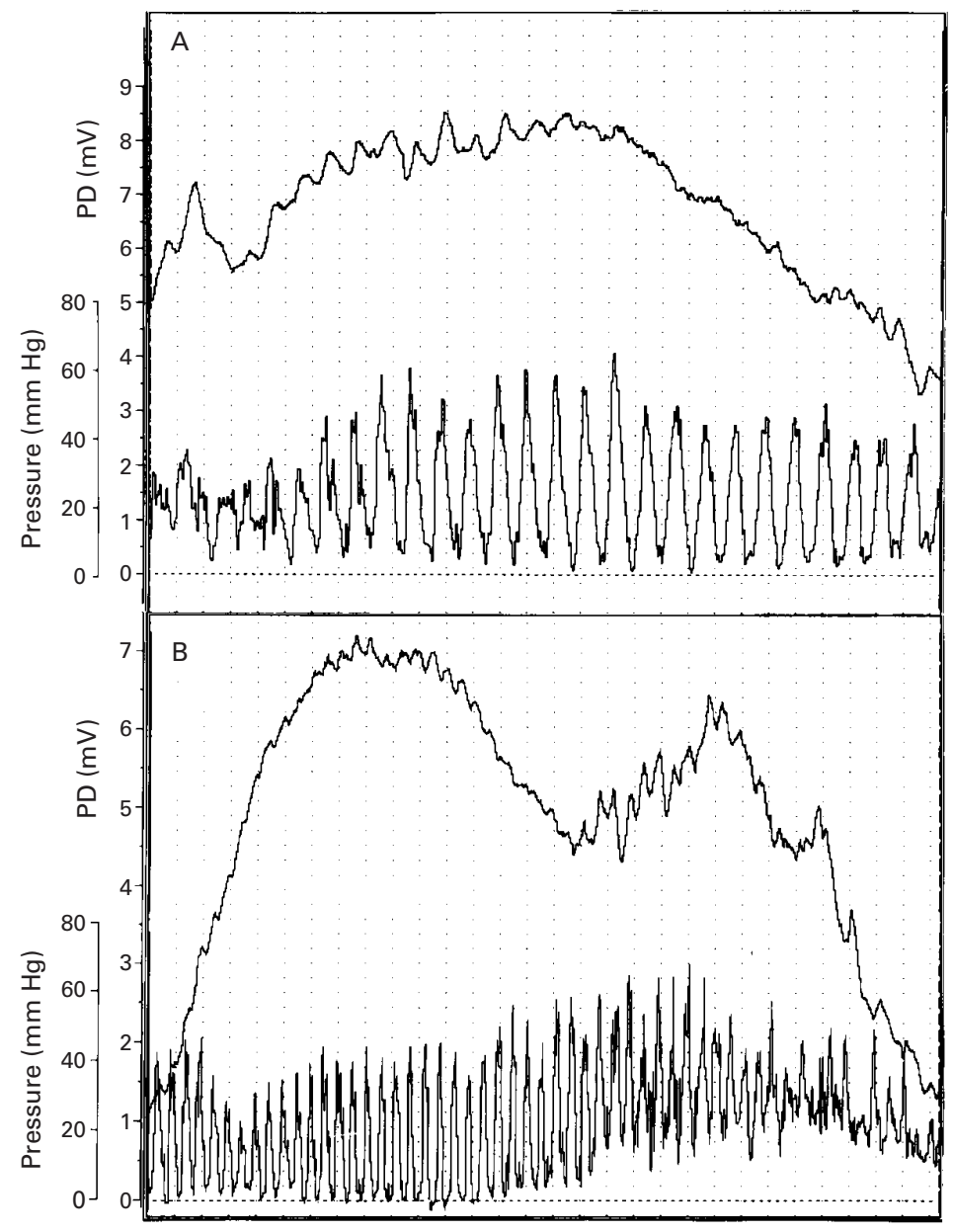

Figure 3 Two original recordings highlighting the escape phenomenon that occurred during phase III. Please note that in this particular figure, the sign of the transmural potential difference (PD) signal is reversed: a higher (positive) PD denotes a more lumen negative PD value. $(A)$ the upper line is the PD signal and the lower line the pressure signal. The distance between the pointed lines is five seconds. The isolated pressure waves during phase III are clearly seen and before the escape phenomenon, each phasic pressure wave is accompanied by an increase in PD of around $0.5 \mathrm{mV}$, without any consistent time delay. After reaching its peak, PD decreases despite continuing motor activity, and in this particular subject, the PD changes induced by isolated contractions were also temporarily abolished. (B) An example of "interruption" of the escape period by a rise in mean pressure. Please note that the time resolution is lower than in the upper panel: the distance between the dotted lines is 10 seconds. In this subject, PD waves (although of smaller magnitude) were also visible during the escape phase. About 80 seconds after the PD peak, $P D$ starts increasing again, and when this happens, there is also a sustained rise in mean pressure.
Analysis of PD changes during sustained phasic motor activity (phase III)

At the start of phase III, duodenal PD started to increase (become more lumen negative) without any measurable time delay (4 (4) s). The initial slope of the PD curve was relatively constant in the different experiments $(-101$ (9) $\mu \mathrm{V} / \mathrm{s}$, range -58 to -142 ). In $13 / 22$ phase III periods, the PD waves associated with the individual contractions were also readily discernible during the rising phase (fig 3A). This figure also illustrates that, with reservations for limitations set by the sampling frequency (4 $\mathrm{Hz}$ ), there was no measurable time delay between the start of the individual contraction and the associated PD wave (fig 3A). During 9/22 phase III periods, the PD waves disappeared during the rising phase.

PD eventually reached a maximal (most negative) value that was relatively constant between experiments (range in whole material -7.5 to $-12.5 \mathrm{mV}$ ). In $3 / 22$ cases, the PD waves disappeared during this peak period. PD then began to fall, despite continuing phasic motor activity. However, in 21/22 cases, individual contractions were still associated with PD waves during this "escape" period. The responsiveness of the system was particularly obvious in one case (fig 3B) in which we saw a sustained increase in mean pressure during the escape period. It can be seen that, during this period, PD again started to rise. This pattern was, however, very uncommon. Mean pressure consistently reached its maximal value after the PD peak (+ 31 (7) seconds, $\mathrm{p}<0.01$ ), and then decreased slowly despite continuing phasic motor activity. The decrease in PD approximately followed a monoexponential curve, with a half time of about two minutes (mean value 110 seconds).

The relation between pressure and PD was grossly similar in the duodenum and jejunum, but there were quantitative differences before, during, and after phase III between the two segments (table 2). During late phase II, luminal pressure was significantly lower in the jejunum. PD was lower (less lumen negative) in the jejunum during both phase I-II and late phase II. The peak pressure, but not the peak PD, was also lower in the jejunum. In both segments, the PD peak occurred before the pressure peak, but the time to reach peak pressure or peak PD values was significantly longer in the jejunum, and this difference was also reflected by a less steep mean slope of the jejunal PD curve. There was no significant correlation between the mean slope of the pressure and PD curves in individual experiments in either the duodenum or the jejunum.

To test if the slope and peak of the phase III related PD increase depends on mucosal secretory function, we also recorded eight phase III periods in three patients with villus atrophy. In the controls $(n=9)$, the slope of the PD curve was $-102(7) \mu \mathrm{V} / \mathrm{s}$ and the peak PD value was $-9.2(0.7) \mathrm{mV}$. In the patients with villus atrophy, both these values were significantly elevated: (slope: $-199 \quad(42) \mu \mathrm{V} / \mathrm{s}$, p<0.01; PD (peak): -17.1 (0.9) mV, p<0.001). 
Table 2 Comparison of phase related pressure and transmural potential difference (PD), simultaneously recorded from duodenum and jejunum in eight subjects

\begin{tabular}{|c|c|c|c|c|}
\hline \multirow[b]{2}{*}{ Period } & \multicolumn{2}{|c|}{ Pressure $(\mathrm{mm} \mathrm{Hg})$} & \multicolumn{2}{|l|}{$P D(m V)$} \\
\hline & Duodenum & fejunum & Duodenum & fejunum \\
\hline Phase I-II & $2.8(0.7)$ & $2.0(0.8)$ & $-2.2(0.2)$ & $-1.2(0.3)^{\star}$ \\
\hline Late phase II & $4.5(1.3)$ & $3.0(0.7)^{\star}$ & $-3.5(0.3)$ & $-2.0(0.4)^{\star}$ \\
\hline \multicolumn{5}{|l|}{ Phase III } \\
\hline Peak value & $32.4(3.1)$ & $20.8(2.3)^{\star}$ & $-9.2(0.3)$ & $-9.3(0.8)$ \\
\hline Time to peak (seconds) & $123(26)$ & $220(20)^{\star}$ & $95 \quad(13)$ & $165(25)^{\star}$ \\
\hline Mean slope (PD) $(\mu \mathrm{V} / \mathrm{s})$ & & & $-76 \quad(7)$ & $-55(7)^{\star}$ \\
\hline
\end{tabular}

Data are mean values during the corresponding periods for phase I-II and late phase II. The phase III peak value is the highest value recorded during phase III (time resolution 10 seconds). The time to peak is the time from phase III onset to the peak value. The mean slope of the PD curve was calculated by linear regression from all points between phase III onset and the PD peak. The upward slope is given as a positive value. Data are mean (SEM).

${ }^{\star} \mathrm{p}<0.05$, significant difference from duodenal values.

RELATION BETWEEN CONTRACTION FREQUENCY, PD, BILE RELEASE, AND NFT DURING A FULL MMC CYCLE

Figure 4 summarises how contraction frequency and PD varied during a full MMC cycle. The perfusate was Krebs/mannitol (see Materials and methods). The time scale is relative (percentage of full cycle length), for reasons given in the methodological section. The least negative PD was obtained during MMC phase I (10-15\% of cycle). A significant increase in PD (more lumen negative) occurred in the $75-80 \%$ interval $(p<0.001)$ and the highest (most lumen negative) PD was attained during phase III (95-100\% interval).

Figure 5A shows the time course of the MMC related changes in contraction frequency and NFT. The same time resolution was used when calculating motility changes and transport changes (15 minutes; see Materials and methods for details on the calculations). When recorded with this relatively low time resolution, the lowest motor activity occurred in the $25-30 \%$ interval and the increase became significant in the $65-70 \%$ interval. The maximal motor activity occurred

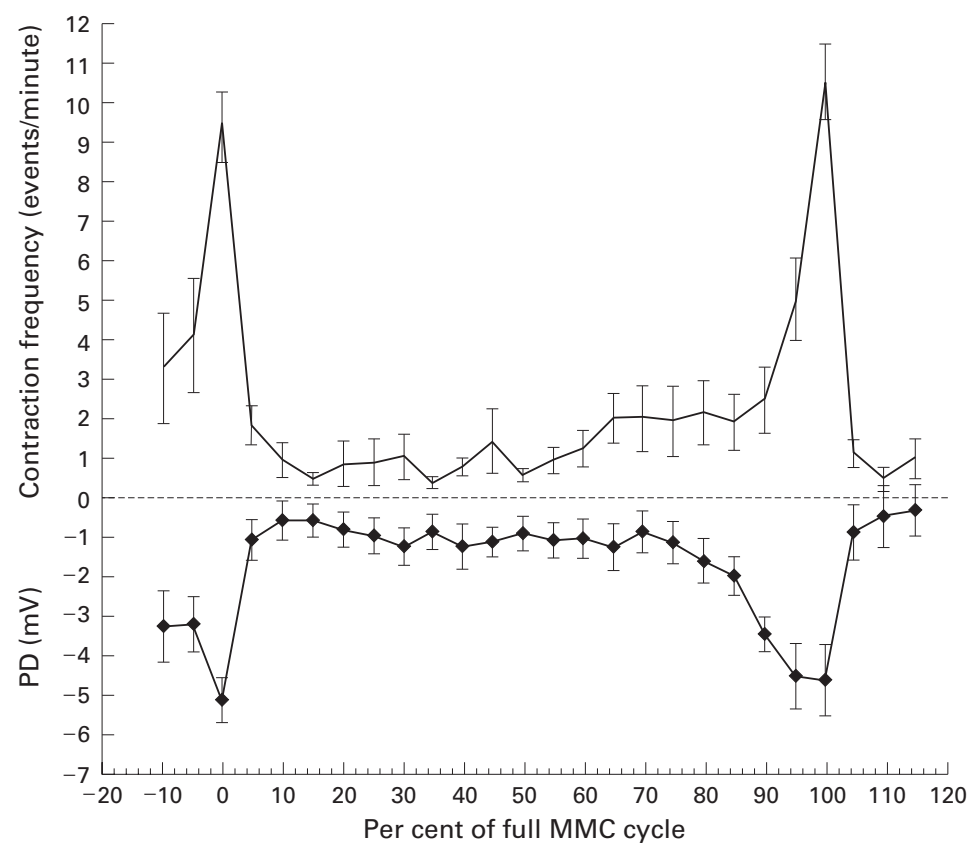

Figure 4 Contraction frequency (upper graph) and transmural potential difference (PD) (lower graph) during a full migrating motor complex (MMC) cycle. The original data (before expression as \% of full MMC cycle) had a time resolution of one minute. Values are mean $(S E)(n=8)$. during the $90-95 \%$ interval. The corresponding curve for NFT showed a maximum absorption in the $20-25 \%$ interval, and the change in the secretory direction also became significant in the $65-70 \%$ interval $(\mathrm{p}<0.01$, non-parametric analysis of variance). However, transport rate then again returned towards control levels before the start of phase III, the change no longer being significant after the $80-85 \%$ interval.

Figure 5B shows the time course of luminal bilirubin release. Absolute release rates varied considerably, and for the sake of clarity, median values only are given. It is seen that luminal bile release also increased during the last half of the cycle. To quantify the relative importance of motor activity and luminal bile, we calculated the predictive value of a 2 SD increase in motor activity and a 2 SD increase in bile release, to detect a 2 SD change in NFT in the secretory direction (see Materials and methods). Motor activity itself had a predictive value of 50 (5)\% and bile release itself a predictive value of 65 (7) $\%$. However, the predictive value of a combination of motor activity and bile release was 79 (5)\%, which was significantly higher than both motor activity only $(\mathrm{p}<0.01)$ and bile release only $(\mathrm{p}<0.05)$. Bile in the lumen thus further increased the probability of fluid secretion.

\section{Discussion}

When measuring over hours, we found a quantitative linkage between low pass filtered pressure and PD changes with an amplification factor of around $0.3 \mathrm{mV} / \mathrm{mm} \mathrm{Hg}$, but isolated contractions only gave rise to very small, apparently amplitude independent, PD waves. During a longer period of repeated lumen occlusive phasic contractions (MMC phase III), PD first rose rapidly to a peak value, at a rate that was higher in the duodenum than in the jejunum and higher in patients with villus atrophy than in controls. PD then returned towards control values, despite continuing phase III motor activity and a seemingly intact PD response to isolated contractions. Surprisingly, the pronounced PD increase during phase III was not accompanied by any measurable change in NFT.

Some of the apparent paradoxes of this pattern may be due to the appreciably different time course of the pressure, PD, and fluid transport signals. The pressure signal is both 

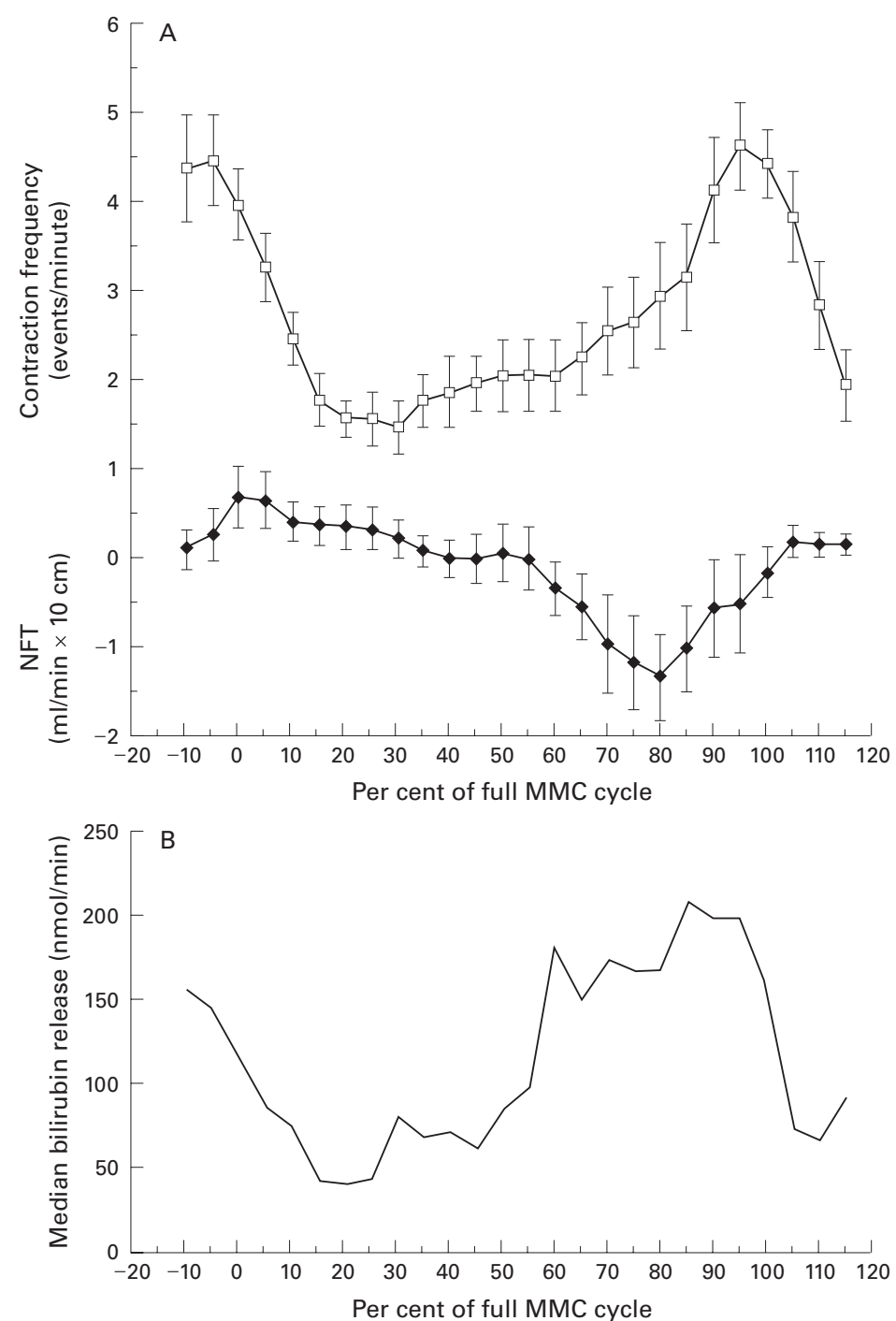

Figure 5 (A) Contraction frequency, net fluid transport (NFT), and (B) bilirubin release, expressed against a common relative time scale (\% of full migrating motor complex $(M M C)$ cycle). The time resolution of the original data was identical for the three variables: 15 minutes. The values in $(A)$ (frequency and net fluid transport are mean (SE) $(n=13)$. In (B) (bile), median values only are given. The unit for bilirubin release was nmol/min.

technically and biologically virtually instantaneous. The PD signal is technically also fast, but biologically slower, as it depends on activation of a secretory membrane process. The water transport signal, finally, ought to be biologically similar to the PD signal, but is technically very slow, as it can only be measured by perfusion techniques based on intermittent sampling, which by necessity gives a poor time resolution.

Phase III is a good starting point for the interpretation, as it gives a sustained pressure increase, which thus eliminates the effects of the slow biological time course of the PD signal. At the start of phase III, PD increased at a rate that varied moderately between subjects. The mean rate of increase was around 0.1 $\mathrm{mV} / \mathrm{s}$. This value was in itself a variable, as it was significantly lower in the jejunum and higher in the patients with villus atrophy. Patients with villus atrophy have a potentiated secretory response to motor activity, ${ }^{39}$ and the steeper slope may therefore reflect a higher secretory capacity of the mucosa.

If one accepts this interpretation, the lack of correlation between amplitudes of phasic contractions and PD deflections is not surprising: in the proximal small intestine, a phasic contraction has a duration of about five seconds (60/slow wave frequency). With a maximal responsiveness of the PD signal of about $100 \mu \mathrm{V} / \mathrm{s}$, one would expect a deflection of $0.5 \mathrm{mV}$, which is close to the observed mean value $(0.36(0.04) \mathrm{mV})$. This interpretation seems to be at variance with previous studies by Read and coworkers, ${ }^{23}$ who described PD waves with an amplitude that exceeded by severalfold that seen during isolated contractions in the present study. However, when the original figures presented in these papers are looked at more closely, the contraction waves associated with these large PD changes invariably had a duration of 20 seconds or more - that is, they probably represented cluster contractions rather than isolated spike potentials. In our own material, we also occasionally saw clusterlike contractions, and they were consistently associated with PD increases in the same range (several millivolts) as that reported in the quoted papers (H Sjövall, unpublished).

To eliminate the effect of the slow time course of the PD response, we low pass filtered both pressure and PD data, and studied their correlation during a full MMC cycle-that is, over hours. We found that the pressure and PD curves thus treated were virtually mirror images of each other, with a mean linkage factor of $-0.33(0.07) \mathrm{mV} / \mathrm{mm} \mathrm{Hg}$. This means that, given sufficient time, a pressure increase of $1 \mathrm{~mm} \mathrm{Hg}$ will make the PD $0.33 \mathrm{mV}$ more lumen negative.

An advantage with phase III is that it leads to a sustained increase in mean pressure which eliminates the effect of the slow time course of secretion. We did see the expected increase in PD at the start of phase III, but after about 100 seconds, PD again started to decrease despite continuing phasic motor activity. In all experiments except one, phasic contractions were still associated with PD waves during the escape period. The intact responsiveness of the system was particularly obvious in one case in which there was a sustained increase in mean pressure during the escape period, which led to a clear cut notch in the PD curve.

There are essentially three types of receptors that may generate this complex pattern: muscular stretch and/or tension receptors, ${ }^{19}$ mucosal "touch" receptors, ${ }^{18}{ }^{28}$ and mucosal chemoreceptors. ${ }^{20}$ The finding of a quantitative relation between pressure and PD over hours strongly supports the concept that a muscular receptor is involved. A receptor population that should be able to convey this type of information is one recently described by Kunze et $a l .{ }^{19}$ These neurones, with their cell bodies in the myenteric plexus, are very slow to adapt, and respond to tension rather than to stretch, as firing is reduced by paralysis of the smooth muscle. ${ }^{19}$ On the other hand, this model is apparently at variance with the finding of See and colleagues ${ }^{8}$ that the pressure-PD linkage 
remains intact after chemical damage to the myenteric plexus with benzyldimethyltetradecylammonium chloride. It should, however, be remembered that neurones may fire unspecifically in response to mechanical deformation, and it remains to be shown that the quantitative motility-secretion linkage remains after myenteric plexus ablation.

The intestinal mucosa also contains mechanoreceptors that respond to tactile stimuli. ${ }^{18}{ }^{28}{ }^{29}$ Some of them seem to have their cell bodies in the submucous plexus ${ }^{28}$ and others in the myenteric plexus. ${ }^{18}$ It is very hard to envisage how a receptor of this type could sense the "slow" changes in mean pressure over hours, but they may nevertheless play an important modulatory role, particularly during phase III. During this period, contractions are lumen occlusive, which should lead to pronounced activation of these receptors by compression. ${ }^{29}$ It has previously been shown that simultaneous activation of touch and tension receptors has a potentiating effect on the ascending excitatory component of the peristaltic reflex, ${ }^{29}$ and prolonged stimulation results in a modality specific run down of the reflex response, ${ }^{30}$ with a time course similar to that of the escape of the PD signal during phase III. In virtually all of our experiments, the PD waves associated with individual contractions remained throughout the escape period, a finding that seems incompatible with run down of the tension receptor. One therefore has to postulate that this phenomenon was due to run down of another receptor, possibly a touch receptor. The details are, however, not clear and need to be studied in a better controlled system.

Mucosal chemoreceptors with their cell bodies in the myenteric plexus respond to acid, base, or fatty acids. ${ }^{20}$ Chemoreceptor activation also potentiates the reflex response to touch or tension receptor activation..$^{30}$ In particular, the fluid transport response had a time course that was strikingly similar to that of luminal bile release, and luminal bile also increased the probability of fluid secretion at a given rate of motor activity. It has previously been shown that the presence of bile in the lumen increases the probability of phase III activity, ${ }^{31}$ supporting a net excitatory effect on enteric neurones also at physiological concentrations. It can therefore by no means be excluded that activation of chemoreceptors by luminal bile may potentiate a primarily mechanosensitive reflex mechanism.

Finally, some comments are warranted on the dissociation between PD and NFT at the very end of the MMC cycle. Theoretically, the formulas behind the triple lumen perfusion technique are based on the prevalence of "steady state conditions". ${ }^{32}$ This assumption is obviously incorrect during phase III itself, and possibly also at the very end of phase II and beginning of phase I. Net transport data obtained in the association with phase III should therefore be interpreted with some caution. In previous studies ${ }^{33}$ with the duodenal triple lumen perfusion technique, we have for this reason excluded the period containing phase III activity from the calculations.

In the proximal small intestine, electrically silent fluid absorption occurs by a villus $\mathrm{Na}^{+} / \mathrm{H}^{+}$ exchanger. ${ }^{21}$ We recently used duodenal bicarbonate absorption to indirectly measure duodenal $\mathrm{Na}^{+} / \mathrm{H}^{+}$exchange activity in humans, and found evidence for tonic cholinergic inhibition of this transporter during the first part of the MMC cycle. ${ }^{21}$ In a recently completed series of experiments, we also found that duodenal bicarbonate uptake was considerably enhanced during periods of high motor activity (late phase II and phase III of MMC), and during these periods, we also saw a significant increase in luminal $\mathrm{PCO}_{2}$ levels. ${ }^{34}$ These data are entirely compatible with increased absorption by $\mathrm{Na}^{+} / \mathrm{H}^{+}$exchange, because of improved stirring of the luminal contents. One possible explanation for the apparent "waning" of the net fluid secretion before the start of phase III may therefore be that the secretory water flux generated by electrogenic chloride secretion was overridden by increased efficiency of electrically silent sodium absorption.

To conclude, our findings confirm and extend the results obtained in the pioneer studies by Read, Greenwood and coworkers. The quantitative data and the current neurophysiological evidence do not support the idea that the linkage is solely mediated by a reflex confined to the mucosa-submucosa. The most simple scheme that accounts for the behaviour of the system is a reflex triggered by a slowly adapting tension receptor, the gain of which is modulated by mucosal touch receptors and possibly also by luminal chemoreceptors. From these observations, it can be predicted that the most potent stimulus for electrogenic secretion is lumen occlusive phasic pressure activity with a duration of less than 100 seconds-that is, shorter than the start of the adaptation phenomenon. Incidentally, exactly this type of pattern, which is called "discrete clusters", has been reported to occur often in patients with both functional dyspepsia and diarrhoea-prone irritable bowel syndrome. ${ }^{15-17}$

This study was supported by the Swedish Medical Research Council (grants 8288 and 10328). The excellent technical Council (grants 8288 and 10328). The excellent technistance of Irmelin Hagman is gratefully acknowledged.
assistan

1 Greenwood B, Davison JS. The relationship between gastrointestinal motility and secretion. Am $\mathcal{f}$ Physiol 1987;252:G1-7

2 Read NW, Smallwood RH, Levin RJ, et al. Relationship between changes in intraluminal pressure and transmural potential difference in the human and canine jejunum in vivo. Gut 1977;18:141-51.

3 Read NW. The migrating motor complex and spontaneous fluctuations of transmural potential difference in the human small intestine. In: Christensen J, ed. Gastrointestinal motility. New York: Raven Press, 1980:299-306.

4 Greenwood B, Diamant S, Davison JS. The relationship between motor activity and transmural potential difference in the guinea pig intestine in vitro: is there a neural link? Can $\mathcal{F}$ Physiol Pharmacol 1986;64:993-8.

5 Greenwood B, Doolittle T, See NA, et al. Effects of substance $\mathrm{P}$ and vasoactive intestinal polypeptide on contractile activity and epithelial transport in the ferret jejunum. Gastroenterology 1990;98:1509-17.

6 Baxter PS, Read NW, Hardcastle PT, et al. Abnormal jejunal potential difference in cystic fibrosis. Lancet 1989:1:464-6.

7 Mellander A, Abrahamsson $\mathrm{H}$, Sï̈vall $\mathrm{H}$. The migrating mand A, Abrahamsson H, Sjovall $H$. The migrating motor complex: the motor component of a cholinergic 154:29-41.

8 See NA, Greenwood B, Bass P. Submucosal plexus alone integrates motor activity and epithelial transport in rat jejunum. Am f Physiol 1990;259:G593-98. 
9 Mellander A, Abrahamsson H, Sjövall H. Duodenal Mellander A, Abrahamsson $H$, Sjovall $H$. Duodenal
secretomotor function in untreated coeliac disease. Scand $\mathcal{F}$
Gastroenterol 1995;30:337-43.

10 Sababi M, Nylander O. Elevation of intraluminal pressure and cyclooxygenase inhibitors increases duodenal alkaline secretion. Am F Physiol 1994;266:G22-30.

11 Frieling T, Wood JD, Cooke HJ. Submucosal reflexes: distension-evoked ion transport in the guinea pig distal colon. Am f Physiol 1992;263:G91-6.

12 Itasaka S, Shiratori K, Takahashi T, et al. Stimulation of intramural secretory reflex by luminal distension pressure in rat distal colon. Am $\mathcal{F}$ Physiol 1992;263:G108-14.

13 Schulzke J-D, Fromm M, Hegel U, et al. Ion transport and enteric nervous system (ENS) in rat rectal colon: mechanical stretch causes electrogenic Cl-secretion via Plexus Meissner and amiloride-sensitive electrogenic Naabsorption is not affected by intramural neurons. Pflügers Arch 1989;414:216-21.

14 Schulzke J-D, Riecken E-O, Fromm M. Distension-induced electrogenic secretion is mediated via VIP-ergic neurons in electrogenic secretion is mediated via VIP-ergic neur

15 David D, Mertz H, Fefer L, et al. Sleep and duodenal motor activity in patients with severe non-ulcer dyspepsia. Gut 1994;35:916-25.

16 Kellow JE, Philips SF. Altered small bowel motility in irritable bowel syndrome is correlated with symptoms. Gastroen terology 1987;92:1885-93.

17 Husebye E. The patterns of small bowel motility: physiology and implications in organic disease and functiona disorders. Neurogastroenterol Motil 1999;11:141-61.

18 Smith TK, Furness JB. Reflex changes in circular muscle activity elicited by stroking the mucosa: an electrophysiological analysis in the isolated guinea-pig ileum. F Auton Nerv Syst 1988;25:205-18.

19 Kunze WAA, Furness JB, Bertrand PP, et al. Intracellular recording from myenteric neurons of the guinea-pig ileum
that respond to stretch. $\mathcal{F}$ Physiol (Lond) 1998;506:827-42.

20 Bertrand PP, Kunze WAA, Bornstein JC, et al. Analysis of the responses of myenteric neurons in the small intestine to the responses of myenteric neurons in the small intestine to
chemical stimulation of the mucosa. Am $\mathcal{F}$ Physiol 1997;273: G422-35.

21 Mellander A, Sjövall H. Indirect evidence for cholinergic inhibition of intestinal bicarbonate absorption in humans. Gut 1999; 44:353-60
22 Harris MS, Dobbins JW, Binder HJ. Augmentation of neutral sodium chloride absorption by increased flow rate in tral sodium chloride absorption by increased
rat ileum in vivo. $\mathcal{F}$ Clin Invest $1986 ; 78: 431-8$.

23 Hydén S. A turbidimetric method for determination of higher polyethylene glycols in biological materials. Annals of the Royal Agricultural College of Sweden 1956;22:139-45.

24 Arndorfer RC, Stef JJ, Dodds WJ, et al. Improved infusion system for esophageal manometry. Gastroenterology 1977; 73:23-7.

25 Husebye E, Skar V, Aalen OO, et al. Digital ambulatory manometry of the small intestine in healthy adults. Estimates of variation within and between individuals and
statistical management of incomplete MMC periods. Dig Dis Sci 1990;35:1057-65.

26 Dooley CP, Di Lorenzo C, Valenzuela JE. Variability of migrating

k J, Fändriks L, Olbe L, et al. Mechanisms behind changes in gastric acid and bicarbonate outputs during the human interdigestive motility cycle. Am f Physiol 1996;270: G113-22.

28 Kirchgessner AL, Tamir H, Gershon MD. Identification and stimulation of intrinsic sensory neurons of the submucosal plexus of the guinea pig gut: activity-induced expression of Fos immunoreactivity. $\mathcal{F}$ Neurosci 1992;12:235-48.

29 Yuan SY, Furness JB, Bornstein JC, et al. Mucosal distortion by compression elicits polarized reflexes and enhances responses of the circular muscle to distension in the small intestine. F Auton Nerv Syst 1991;35:219-26.

30 Smith TK, Bornstein JC, Furness JB. Interactions between reflexes evoked by distension and mucosal stimulation: electrophysiological studies of guinea-pig ileum. F Auton Nerv Syst 1991;34:69-76.

31 Hellström P, Nilsson I, Svenberg T. The role of bile in the regulation of gut motility. F Intern Med 1995;237:395-402.

32 Cooper H, Levitan R, Fordtran IS, et al. A method for studying absorption of water and solute from the human studying absorption of water and solute from

33 Sjövall H, Hagman I, Abrahamsson H. Relationship between interdigestive duodenal motility and fluid transport in humans. Am f Physiol 1990;259: G348-54.

34 Mellander A, Hagman M, Sjövall H. Effects of motor activity on active bicarbonate absorption in human duodenum [abstract]. Neurogastroenterol Motil 1998;10:87. 